\title{
NXS - a program library for neutron cross section calculations
}

\author{
MIRKO BOIN ${ }^{a *}$ \\ ${ }^{a}$ Helmholtz Centre Berlin, Hahn-Meitner-Platz 1, 14109 Berlin, Germany. \\ E-mail: boin@helmholtz-berlin.de \\ (Received 0 XXXXXXX 0000; accepted 0 XXXXXXX 0000)
}

\begin{abstract}
A collection of routines calculating neutron scattering and absorption cross sections on the basis of crystal structure descriptions is presented and implemented into the new and reusable $n x s$ program library. An example program providing a graphical user interface to the nxs functions is created to demonstrate their usage. The flexibility of the library and the possibilities for multiple areas of application are shown by further examples involving Monte Carlo neutron simulations concerned with imaging experiment validation and neutron instrument development.
\end{abstract}

\section{Motivation}

Neutrons have proven to be a powerful probe for the exploration of structural and dynamical properties of solids and liquids. In order to satisfy the growing demand for neutron beam time new materials, components and techniques are continuously being developed to be used at reactors and spallation sources for the production, thermalisation and transport of neutrons respectively. Ongoing optimisations of neutron 
sources and moderators, for example, require reliable cross section data of materials comparable to those that are considered to be used in such environments (Gillette et al., 1999) to estimate the neutron scattering and absorption behaviour. Likewise, instrument upgrades in order to achieve more neutron flux and higher resolution at the sample position can only be realised if the individual instrument components are well characterised. Such different setups can be evaluated in advance. However, in order to use neutron beam time effectively careful experiment preparation is just as important. In general, the calculation of expected spectra would help to save beam time if measurement positions of interest can be selected beforehand. Known cross section values could be used in refinement and fitting routines to achieve consistent results. Moreover, a comparison of different computed sample characteristics may help to analyse and validate the performed experiment. If the conditions of an existing neutron instrument are simulated the above mentioned experiment preparation and evaluation can further be improved. Then, the discussion on the feasibility of a measurement and the validity of the analysed results are supported by the results of the virtual experiment.

Besides the support in experimental data analysis, Monte Carlo simulations have become more and more important for the development, e.g. the design and upgrade phase, of neutron instruments (Šaroun et al., 2002). The complexity of the instrument is handled by a large number of parameters describing the experimental conditions such that multiple scenarios can be tested before. Similarly, the count rate to achieve the required signal quality can be estimated with the help of such simulations. Consequently, a prediction of the necessary exposure time is possible if a virtual sample is accurately described by means of its material composition. For this purpose, the sample's crystal structure and the arrangement of atoms inside the unit cell are applied in this work for the calculation of neutron cross sections.

IUCr macros version 2.1.4: 2010/12/07 


\section{Neutron cross section calculations}

Neutron-matter interaction is described quantitatively by neutron cross sections $\sigma$ which depend on the material properties as well as on the neutron wavelength $\lambda$ (Jevremovic, 2008). Neutron cross sections of a chemical element are given in barns $\left(1\right.$ barn $\left.=10^{-24} \mathrm{~cm}^{2}\right)$ and refer to an effective area representing a measure of scattering and absorption probabilities for an incident neutron. The following part gives an overview of the cross section calculations, presented by Granada (1984) and Vogel (2000). It shall be noted that the magnetic interaction is neglected here. Hence, the term total neutron cross section will be described as the summation of the individual coherent, incoherent, elastic, inelastic and absorption contributions.

$$
\begin{aligned}
\sigma_{\text {total }}(\lambda)= & \bar{\sigma}_{\text {coh }}\left(S_{c o h}^{e l}(\lambda)+S_{c o h}^{\text {inel }}(\lambda)\right) \\
& +\bar{\sigma}_{\text {inc }}\left(S_{\text {inc }}^{\text {el }}(\lambda)+S_{\text {inc }}^{\text {inel }}(\lambda)\right)+\sigma_{a b s}(\lambda)
\end{aligned}
$$

The scattering functions $S$ describe the neutron wavelength influence and the effect of the spatial arrangement of nuclei, i.e. crystal structure, on the cross sections. $\bar{\sigma}_{c o h}$ and $\bar{\sigma}_{\text {inc }}$ are calculated using the scattering length $b$, the atom concentration $c_{n}$ and the nuclear constant $\sigma_{i n c, n}$ of the contributing nuclei $n$ :

$$
\begin{aligned}
\bar{\sigma}_{c o h} & =4 \pi\left(\sum_{n} c_{n} b_{n}\right)^{2} \\
\bar{\sigma}_{i n c} & =4 \pi\left(\left(\sum_{n} c_{n} b_{n}^{2}\right)-\left(\sum_{n} c_{n} b_{n}\right)^{2}\right)+\sum_{n} c_{n} \sigma_{i n c, n} \\
\sigma_{a b s} & =\sum_{n} \sigma_{a b s, n}(\lambda)=\sum_{n} B_{n} \frac{1}{v}=B_{n} \frac{m \lambda}{h}=\frac{\sigma_{a b s, n}^{2200}}{1.798 \AA} \lambda
\end{aligned}
$$

The absorption cross section $\sigma_{a b s}$ for a complete unit cell is the sum of the individual atom contributions $n$ which are determined by means of the neutron velocity $v$ and a proportionality factor $B_{n}$. This dependency results from the fact that the longer a neutron stays in the environment of a target nucleus the greater the chance it will be absorbed (Windsor, 1981). Most reference absorption cross sections are available 
in terms of a standard velocity of $v=2200 \mathrm{~ms}^{-1}$, which equates a wavelength of $\lambda=1.798 \AA$. Thus, the proportionality of $1 / v$ can be transformed into a wavelength dependence to determine $\sigma_{a b s, n}(\lambda)$.

The structural characteristics of the material are considered in the description of the coherent elastic scattering cross section (Fermi et al., 1947) by means of the structure factor $F$ and the spacing $d$ of selected $(h k l)$ lattice planes and the unit cell volume $V_{0}$ :

$$
\sigma_{c o h}^{e l}(\lambda)=\bar{\sigma}_{c o h} S_{c o h}^{e l}(\lambda)=\frac{\lambda^{2}}{2 V_{0}} \sum_{d_{h k l}=0}^{2 d_{h k l}<\lambda}\left|F_{h k l}\right|^{2} d_{h k l}
$$

The fraction of neutrons that scatter elastically also includes incoherent scattering whose wavelength-dependent definition $S_{i n c}^{e l}$ is based on the assumption of thermal motion of the nuclei. Therefore, an isotropic atomic displacement factor $B_{i s o}$, also known as the Debye-Waller factor (Debye, 1913 \& Waller, 1923) was introduced to compute the incoherent part. For a calculation of multi-atomic systems, the contributions are added for each atom $n$ inside the unit cell as the incoherent elastic part $\sigma_{i n c}^{e l}$ does not depend on the crystal structure:

$$
\begin{aligned}
S_{i n c}^{e l}(\lambda) & =\frac{\lambda^{2}}{2 B_{i s o, n}}\left(1-\exp \left(-\frac{2 B_{i s o, n}}{\lambda^{2}}\right)\right) \\
\sigma_{i n c}^{e l}(\lambda) & =\bar{\sigma}_{i n c} \sum_{n} S_{i n c}^{e l}(\lambda)
\end{aligned}
$$

The inelastic contributions to the total neutron cross section calculation describe the probabilities for a neutron to lose or gain energy when interacting with the crystal lattice. It was shown in (Binder, 1970) that the incoherent part of the inelastic scattering cross section is a good approximation for the coherent part in the thermal neutron range. Hence, the total inelastic scattering cross section can be described by:

$$
\sigma_{\text {total }}^{\text {inel }}(\lambda)=\sigma_{\text {coh }}^{\text {inel }}(\lambda)+\sigma_{\text {inc }}^{\text {inel }}(\lambda)=\left(\bar{\sigma}_{\text {coh }}+\bar{\sigma}_{\text {inc }}\right) S_{\text {inc }}^{\text {inel }}(\lambda)
$$

In order to determine $S_{i n c}^{\text {inel }}(\lambda)$, a procedure proposed by (Granada, 1984) is used. At first the incoherent elastic scattering contribution $S_{i n c}^{e l}(\lambda)$, i.e. equation (4), is IUCr macros version 2.1.4: 2010/12/07 
evaluated. Then, the total incoherent part is calculated, which is given by a rapidly converging mass expansion (Placzek, 1954) and approximated by:

$$
S_{\text {inc }}^{\text {total }}(\lambda)=\left(\frac{M / m}{M / m+1}\right)^{2}\left(1+\frac{9 m^{2} \varphi_{3}(\theta) \varphi_{1}(\theta) \lambda^{2}}{2 M^{2} B_{i s o, n}}\right)
$$

where $m$ and $M$ are the neutron and the scattering nucleus mass respectively. $\varphi_{1}$ and $\varphi_{3}$ are functions of $\theta=\frac{T}{\theta_{D}}$, being the ratio between sample and Debye temperature. Their definitions and evaluations are reported in detail by Granada (1984). The inelastic part is calculated for each contributing nucleus to obtain the total inelastic scattering cross section for a unit cell. Additionally, one is now able to apply the approximation given in equation (5) in order compute the coherent inelastic part individually.

\section{The nxsPlotter example program}

The above presented calculations have been implemented using the $\mathrm{C}$ programming language. The implementation has been compiled into the new and flexible neutron cross section library, called $n x s$, in order to support and provide a wide range of applications. For example, a standalone program was created to plot wavelength-dependent neutron cross sections and thereby demonstrate the usage of the library routines. In Fig. 1, screenshots of this new program, called nxsPlotter, show the main user interface as well as tabulated computation results. The developed software is based on the Qt4 C++ class libraries (Blanchette \& Summerfield, 2008) for programming graphical user interfaces (GUI). However, also other interfaces and programming languages are conceivable which allow native $\mathrm{C}$ code calls. Moreover, the program code can be compiled and executed on multiple platforms (e.g. Linux, MacOS, Windows), which extends the availability of the library even more. Further applications utilising the $n x s$ library will be presented in section 4 .

IUCr macros version 2.1.4: 2010/12/07 


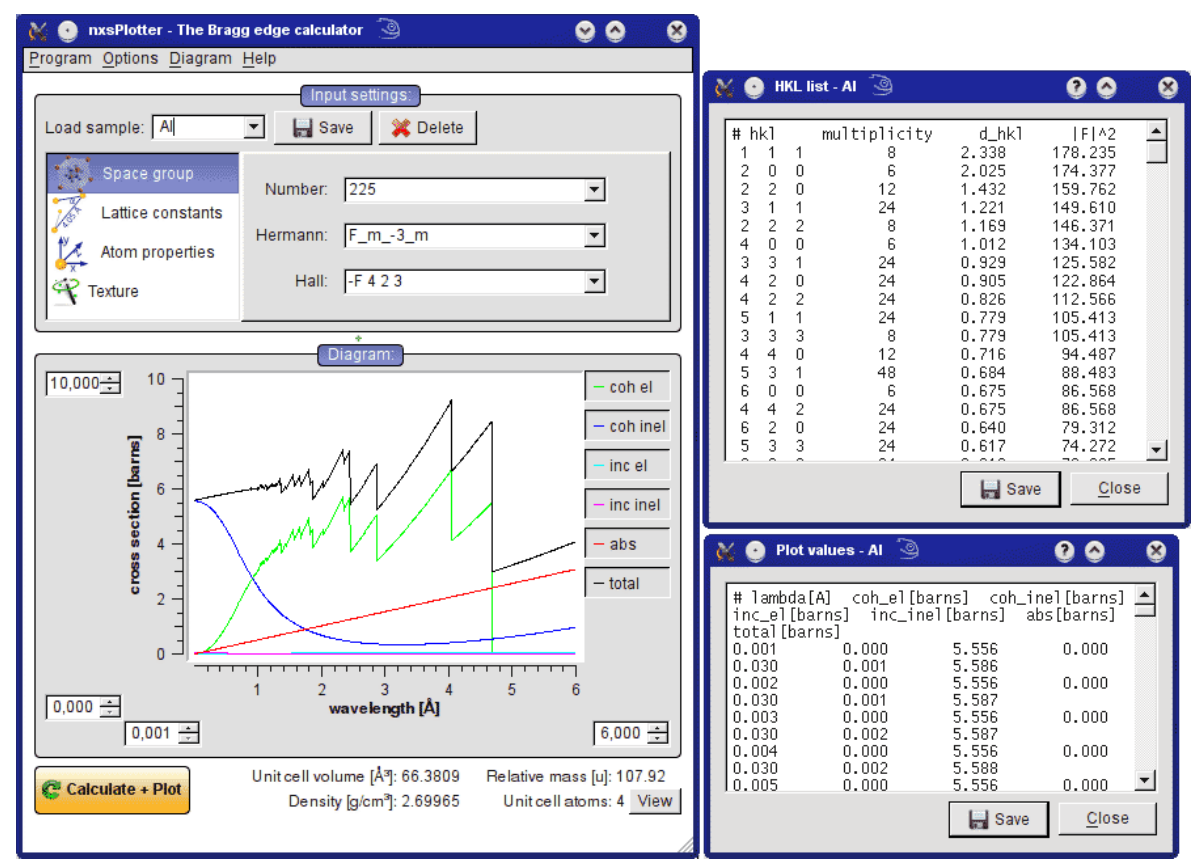

Fig. 1. Screenshots of the nxsPlotter program providing a graphical user interface for the nxs library routines. Left: Main GUI showing the cross section spectrum of an aluminium sample. Right: Tabulated computation results of multiplicities, lattice spacings and structure factors squared for the (hkl) lattice planes and neutron cross section values.

\subsection{Input parameters}

The parameters required for the neutron cross section calculations reviewed above can be summarised by the incident neutron wavelength $\lambda$ and the sample's material composition, which is characterised by its crystal structure definition. For this purpose, the existing and freely available ANSI C SgInfo library routines by (Grosse-Kunstleve, 1995) have been utilised in order to handle space group symmetries. The SgInfo source files have been included in the nxs library as proposed in the project documentation (available at http://cci.lbl.gov/sginfo/). The crystal structure information is obtained from the space group number, or alternatively from a Schönflies or Hermann-Mauguin symbol, which is given as an input parameter. Then, the crystal symmetry information (e.g. cubic, hexagonal or orthorhombic) can be obtained, which requires the lattice 
parameters $a, b, c(\AA)$ and $\alpha, \beta, \gamma\left(^{\circ}\right)$ to be assigned to the unit cell. The sample's material composition is defined by individual atoms and their properties - the atomic mass $M\left(\mathrm{u} \approx 1.66 \times 10^{-27} \mathrm{~kg}\right)$ and their variable placement $(x, y, z)$ to compute the free atom positions of the site symmetries inside the unit cell (Wyckoff, 1930). The corresponding scattering lengths $\left(b_{c o h}\right)$ and cross sections $\left(\sigma_{a b s}^{2200}, \sigma_{i n c}\right)$, given in fm and barns respectively, can be obtained from databases (e.g. Sears, 1992). In nxsPlotter, the parameters are passed to the library routines via input fields.

\subsection{Interface functions}

The principle cycle of the neutron cross section calculation using the nxs library is shown in Fig. 2. At first, a space group is assigned to a $\mathrm{C}$ struct called UnitCell to initialise a unit cell with the help of the included SgInfo routines. Here, the lattice constants are applied and the unit cell volume $V_{0}$ is determined. The second step is to add atoms to the unit cell. Therefore, an AtomInfo struct and the addAtomInfo function were created to store the atomic properties and Wyckoff position of one or more atoms contributing to the unit cell. Finally, the $(h k l)$ lattice planes will be initialised (initHKL function) according to the above defined space group and unit cell parameters. As a last step during this initialisation process, the individual lattice spacings $d_{h k l}$ and the structure factors $F_{h k l}$ will be computed. Subsequently, a user is able to call the nxs functions for the individual cross section calculation as functions of the neutron wavelength $\lambda$. In the nxsPlotter program, this whole procedure is repeated for a number of wavelengths by a single click on the "Calculate + Plot" button, such that a spectrum appears (see Fig. 1).

IUCr macros version 2.1.4: 2010/12/07 


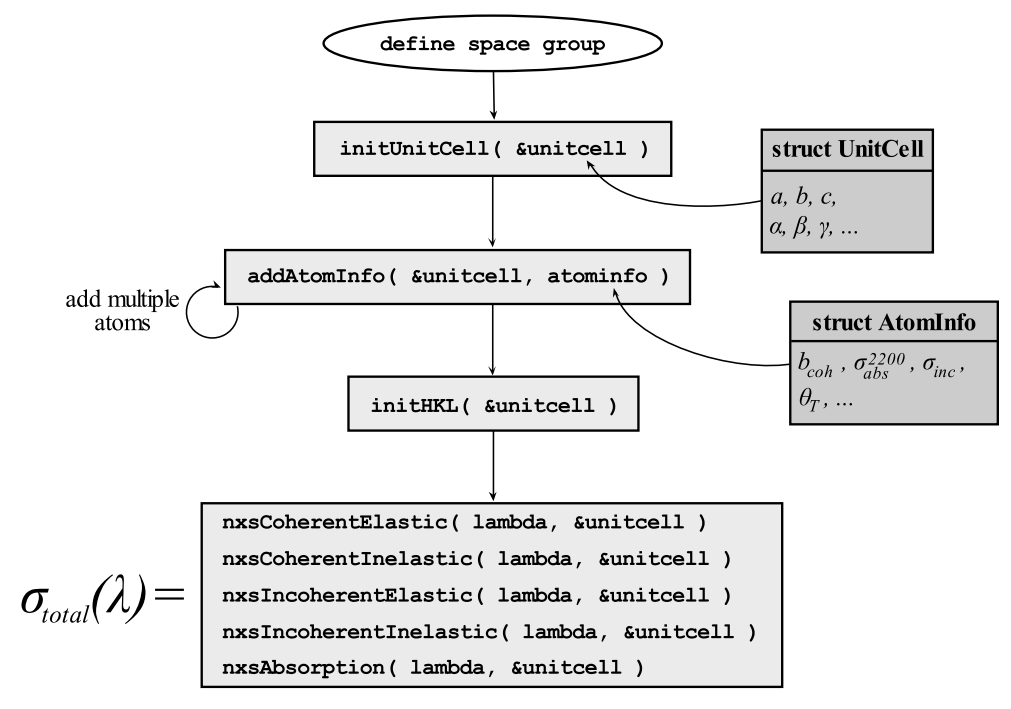

Fig. 2. Cycle to compute wavelength-dependent neutron cross sections with the interface functions of the $n x s$ library.

\subsection{Validation by example}

The implementation of the neutron cross section calculation has been validated with existing tools. The output of nxsPlotter was compared with the values computed by the BETMAn program for Rietveld refinements of transmission data from (Vogel, 2000) and the CRIPO code by (Kropff \& Granada, 1977), a command line tool to calculate neutron cross sections of monoatomic systems only. The latter is able to differentiate between cubic (bcc and fcc) and hexagonal structures, but complex multi-atomic arrangements are not considered. For a comparison with the nxsPlotter results, the values have been normalised by the number of unit cell atoms since $C R I P O$ calculates neutron cross sections per atom. Taking these conditions into account, both results are identical. Additionally, the cross section spectrum for a complex crystal structure, i.e. multiple atoms, has been compared with the output of BETMAn, which is provided with example files. Thus, a $\mathrm{NaCl}$ crystal structure definition was used to validate the $n x s$ calculation. In Fig. 3, the total neutron cross section spectrum of $\mathrm{NaCl}$ computed 
by BETMAn is compared with the nxs results. Details of the material composition are shown in the inset table. It is evident that both the nxsPlotter and the BETMAn results agree, which verifies the present implementation.

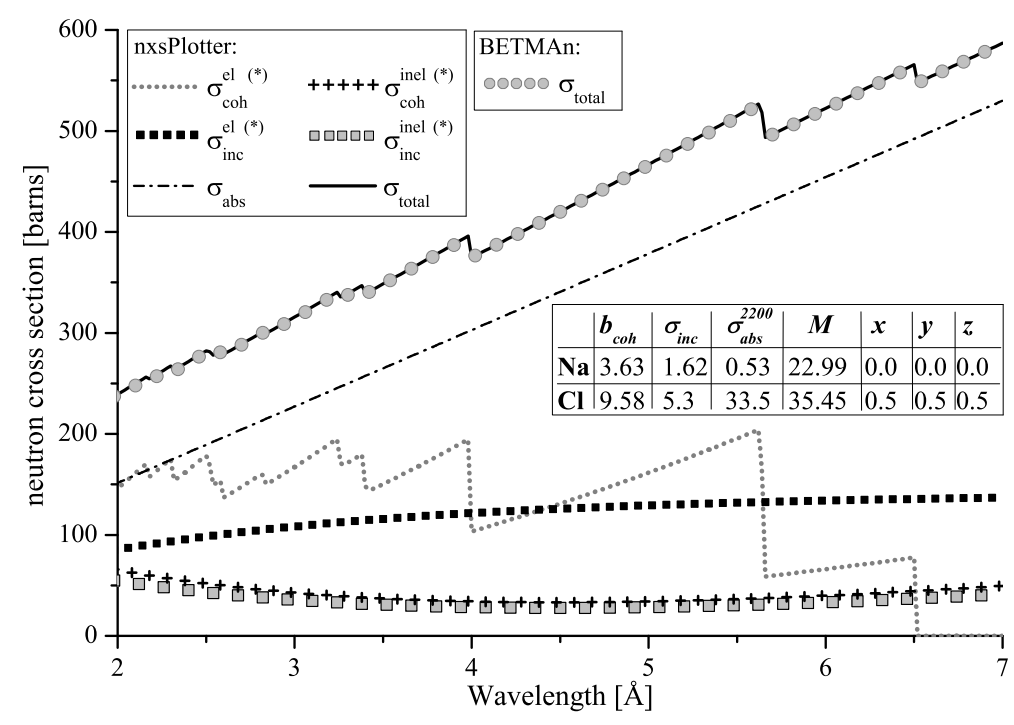

Fig. 3. Comparison of the neutron cross section calculation in nxsPlotter and BETMAn by means of a $\mathrm{NaCl}$ crystal structure. The asterisk $\left(^{*}\right)$ indicates that the individual cross sections have been scaled by a factor of 4 for reasons of clarity.

\section{Application examples}

The above developed GUI provides an example of possible applications for the usage of the nxs routines. In the following, further nxs examples concerned with neutron imaging applications are presented. All of them take advantage of the sudden decreases of the neutron total cross section at defined wavelengths, visible in the plot of Fig. 1 for example, which are known as Bragg edges (e.g. Priesmeyer et al., 1999). They become visible in the neutron transmission spectrum $\operatorname{Tr}(\lambda)$ of polycrystalline samples with thickness $l$ due to the relation between macroscopic cross section, i.e. the attenuation coefficient $\mu$, and $\sigma_{\text {total }} . N$ is the number of scattering centres per unit volume.

$$
\mu(\lambda)=-\ln (\operatorname{Tr}(\lambda)) / l=N \sigma_{\text {total }}(\lambda)
$$




\subsection{Experiment planning and data analysis}

Including a calculation of the attenuation coefficients and therefore of the transmission spectrum, one is able determine the appropriate measurement steps in advance if the sample's material is known. The prediction osf expected intensity variations and Bragg edge positions and magnitudes by means of the developed nxs routines helps to prepare the experiment. Moreover, limitations can be defined in order to save beam time, i.e. if a small wavelength range and/or a single Bragg edge is focussed nxsPlotter supports the choice of the wavelength selection.

In order to improve the precisison of an edge position determination it might further be useful to find the optimal configuration for the sample thickness. Depending on the transmission path length through the sample and the strength of the absorption cross section the amplitudes of the Bragg edges in the transmission spectrum might be diminished by the overall transmission or absorption respectively. However, the height of a Bragg edge, i.e. the difference between the left and the right side of the edge, can be maximised. Santisteban et al. (2001) derived a function to calculate the optimal sample thickness towards the minimisation of the edge position uncertainty which is inversely proportional to the edge height. Therefore, the neutron cross section values on both sides of the Bragg edge must be known. Consequently, whenever it is possible to prepare a sample before a measurement, such that the investigated sample properties will not get affected, the nxs routines assist in achieving the best Bragg edge position resolution by defining an appropriate sample thickness.

Taking advantage of the Bragg edges, structural information about the sample can be obtained from the transmission spectrum, whose analysis is supported by computed spectra. The nxsPlotter GUI, for example, provides different sample characteristics which are easily tested by comparison with the measured results.

IUCr macros version 2.1.4: 2010/12/07 


\subsection{Experiment simulations}

A Monte Carlo computer simulation of different existing Bragg edge imaging neutron instruments has been developed. The McStas neutron ray-tracing software package (Lefmann \& Nielsen, 1999) has been utilised to simulate neutrons propagating through an instrument definition until they have reached a detector. Besides the instrumental conditions, including the source characteristics, guide systems and slits for example, a new sample module has been developed. It takes advantage of the nxs library routines and is therefore able to simulate Bragg edge transmission spectra based on the input parameters described above.

The CONRAD tomography instrument at the Helmholtz Centre Berlin (HZB), utilising a double monochromator device (Treimer et al., 2006) for the selection of single wavelengths from the continuous cold neutron spectrum, and the ENGIN-X diffractometer, equipped with a $10 \times 10$ array of time-of-flight transmission detectors at the ISIS pulsed spallation source, have been simulated for studies of a fibre-textured aluminium sample (Boin et al., 2011). For both instruments, the new sample module, including the nxs library, has been applied to simulate the specimen's transmission spectrum with respect to the instrument parameters. The nxs library was extended with the March-Dollase model (Dollase, 1986), to include a Bragg edge amplitude correction if the sample reveals preferred crystallographic orientations, i.e. texture. Fig. 4 shows the transmission spectra under a defined sample orientation measured on CONRAD and ENGIN-X in comparison to the Bragg edges of texture-free material and the applied simulations. A photograph of the specimen is shown as an inset. The influence of preferred crystallographic orientations on the shape of a Bragg edge is clearly visible. However, the simulations reproduced the experimental results and justified the differences between the results of the two measurements with the individual instrument parameters.

IUCr macros version 2.1.4: 2010/12/07 
A second example, applying the Bragg edge simulation, has been utilised in a proofof-principle study of (Strobl et al., 2011). Bragg edge shifts due to residual stresses in dieless-drawn steel rods were investigated and compared with reference neutron diffraction data. Similar investigations of a standard shrink-fit ring and plug aluminium cylinder have been presented in (Boin et al., 2012) to discuss the feasibility of such measurements for different types of instruments. Both studies made use of the reference data and implemented strain in terms of local lattice spacing variations by modifying the developed nxs sample module. Thus, the measured strain in the Bragg edge transmission direction could be validated with the McStas simulations.

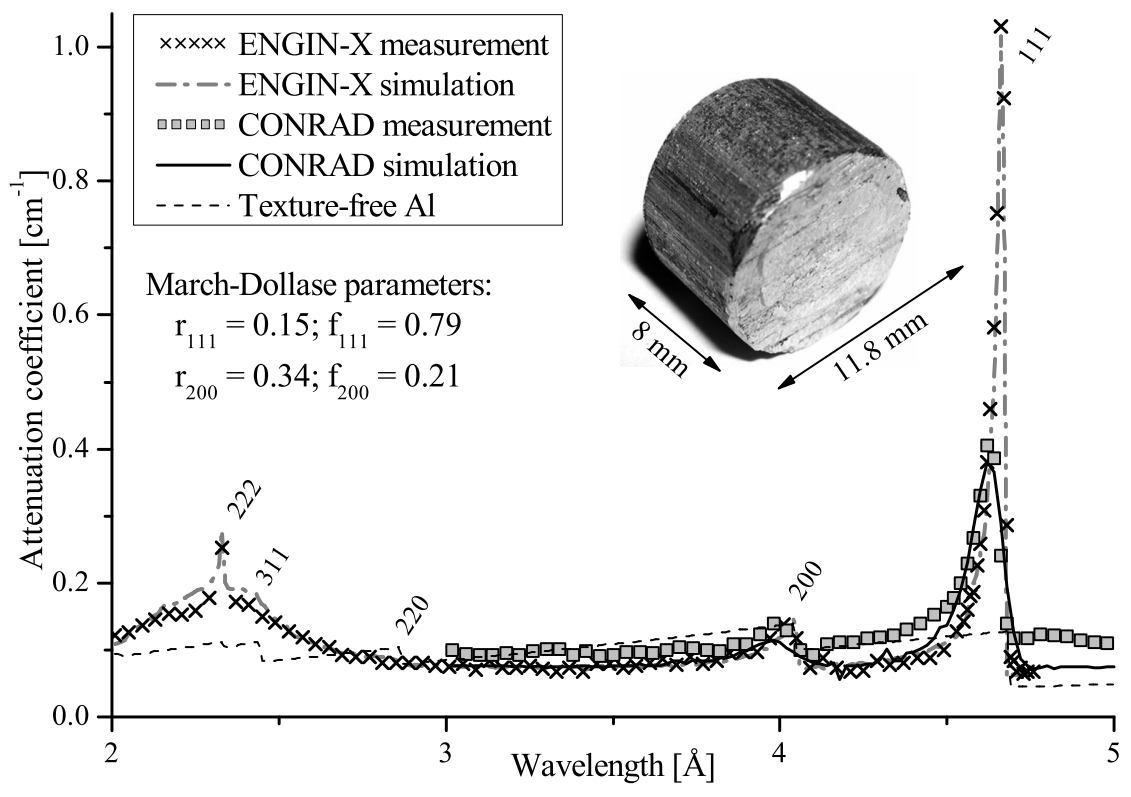

Fig. 4. Measurements and March-Dollase simulations of a textured Al cylinder sample for different neutron instruments.

\subsection{Neutron instrument development}

The developed nxs sample simulation module was further applied within the design process of a new imaging beamline to be built at the ISIS second target station (TS-II) with a repetition rate of $10 \mathrm{~Hz}$. This so-called IMAT instrument will combine neutron 
diffraction and imaging modes for the investigation of engineering materials (Burca et al., 2011). One of the key issues in the design phase is the decision for the position of the new instrument and therefore the choice for the appropriate moderator with respect to the instrument specifications. The performance of the available ports at the east (E7) and west side (W2, W5, W8) of TS-II was benchmarked against the $\mathrm{CH}_{4}$ moderator at TS-I, which operates at four times the repetition rate of TS-II. Hence, a $10 \mathrm{~Hz}$ and $40 \mathrm{~Hz}$ setting of TS-I has been simulated to represent a worst case and best case scenario (Oliver \& Kockelmann, 2008). Fig. 5 shows transmission spectra of a 15-mm thick iron (bcc) sample at different beam ports considered for a final IMAT beamline position simulated with the new McStas module. It is apparent that W8 delivers the highest neutron flux, but at W5, which provides almost the same flux, a longer flight path can be accommodated. Then, the beamline can be placed outside the experimental hall, which is beneficial for the available space at the sample position for example. Accordingly, IMAT will be built at this position $56 \mathrm{~m}$ away from the moderator and outside the TS-II main building.

Similarly, a new imaging instrument is planned at the new European Spallation Source (ESS) which will be built in Lund, Sweden. Multiple neutron imaging techniques are considered to be provided to future instrument users (Strobl, 2009). Therefore, a number of parameters need to be defined in advance in order to design the individual instrument parts according to the specified requirements, e.g. wavelength band and resolution. The HZB simulation group has already started to optimise these parameters using the VITESS neutron instrument simulation software (Lieutenant et $a l .$, 2004). In order to estimate the performance towards Bragg edge imaging applications, for example, a similar sample module as the developed McStas component will be required for the design process of the new ESS instrument. A development of such a module for VITESS is already underway, taking advantage of its $\mathrm{C}$ core such that 
the nxs library routines can be used without any changes.

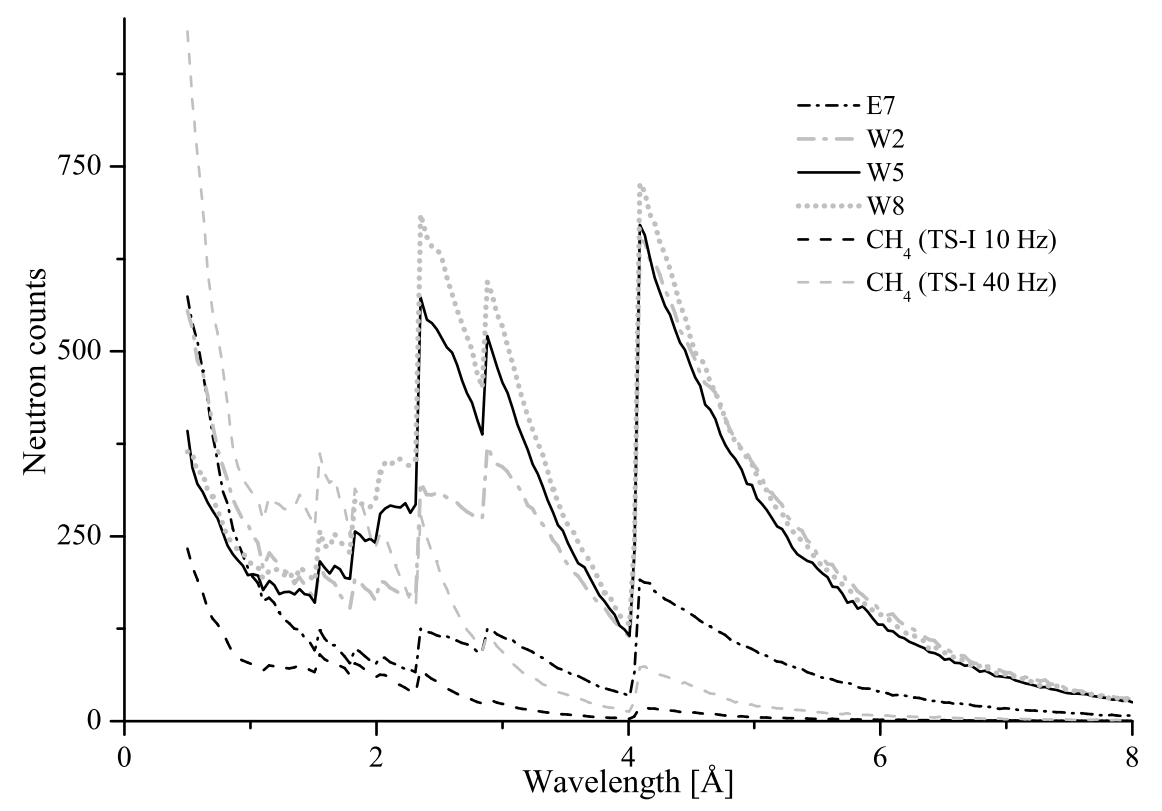

Fig. 5. Simulated transmission spectra of a 15-mm thick iron (bcc) sample at different possible beam ports for the new IMAT beamline position at TS-II benchmarked against the $\mathrm{CH}_{4}$ moderator at TS-I.

\section{Summary}

The $n x s$ library implements the presented neutron cross section calculations for polycrystalline materials that are described by its crystal structure. Thus, the library routines exceed the functionality of the $C R I P O$ code, for example. The nxs program suite is able to deal with all 230 space groups. Nevertheless, the implemented algorithms contain approximations, as stated for equation (5). Furthermore, the Debye temperature model is valid for materials with big atomic masses such as solids, but not for light material such as hydrogen and deuterium. As a consequence, the neutron cross section calculation described here is not reliable for samples with a large fraction of hydrogen, for example. Moreover, the quality of the mass expansion in equation (6) for other than monoatomic systems is not known (Vogel, 2000). Though, IUCr macros version 2.1.4: 2010/12/07 
for engineering neutron diffraction and imaging applications, the $n x s$ implementation is considered as a powerful tool for the presented studies.

Some of the basic calculations are also available in existing tools, e.g. BETMAn, which are not reusable though for the applications shown in this paper. Hence, the neutron cross section algorithms have been re-written with the potential to easily develop new programs and support existing packages, such as the simulation software McStas and VITESS. Both possibilities have been proven by a new stand-alone program to predict neutron cross section spectra and a simulation module for neutron imaging applications and instrument developments respectively. The latter further enables crystal structure definitions and texture and strain parameters to be used with neutron simulation tools for the first time. Besides the imaging part, i.e. neutron transmission, the created sample component is able to handle neutron scattering in general, which will be a beneficial module for realistic experiment simulations particularly with regard to the new upcoming major 2.0 release of McStas. As stated, the same will also be realised with VITESS for the ESS instrument development. Currently, the nxs library, the nxsPlotter program and the simulation modules are available on request from the author.

\section{References}

Binder, K. (1970). Phys. Status Solidi B 41. 767-779.

Blanchette, J. \& Summerfield, M. (2008). C++ GUI Programming with Qt 4. USA: Prentice Hall.

Boin, M.; Hilger, A.; Kardjilov, N.; Zhang, S. Y.; Oliver, E. C.; James, J. A.; Randau, C. \& Wimpory, R. C. (2011). J. Appl. Cryst. 44. 1040-1046.

Boin, M.; Wimpory, R. C.; Hilger, A.; Kardjilov, N.; Zhang, S. Y. \& Strobl, M. (2012). J. Phys: Conf. Ser. 340. 012022.

Burca, G.; James, J. A.; Kockelmann, W.; Fitzpatrick, M. E.; Zhang, S. Y.; Hovind, J. \& Langh, R. van (2011). Nucl. Instrum. Methods Phys. Res., Sect. A 651. 229-235. 
Debye, P. (1913). Ann. Phys. 348. 49-92.

Dollase, W. A. (1986). J. Appl. Cryst. 19. 267-272.

Fermi, E.; Sturm, W. J. \& Sachs, R. G. (1947). Phys. Rev. 71. 589-594.

Gillette, V. H.; Sbaffoni, M. M.; Granada, J. R. \& Pepe, M. E. (1999). Ann. Nucl. Energy 26. $1167-1181$.

Granada, J. R. (1984). Z. Naturforsch. A 39. 1160-1167.

Grosse-Kunstleve, R. W. (1995). SgInfo - a Comprehensive Collection of ANSI C Routines for the Handling of Space Groups. http://cci.lbl.gov/sginfo/.

Jevremovic, T. (2008). Nuclear Principles in Engineering. Boston, MA: Springer US.

Kropff, F. \& Granada, J. R. (1977). CRIPO: A fast computer code for the evaluation of $\sigma_{\text {total }}$ in polycrystalline materials. Unpublished report CAB. Institute Balseiro, Bariloche.

Lefmann, K. \& Nielsen, K. (1999). Neutron News 10. 20-23.

Lieutenant, K.; Zsigmond, G.; Manoshin, S.; Fromme, M.; Bordallo, H. N.; Champion, D.; Peters, J. \& Mezei, F. (2004). In: Sanchez del Rio M. [Ed.]: Avances in Computational Methods for X-ray and Neutron Optics: 3-5 August 2004, Denver, Colorado, USA. Bellingham, Wash.: SPIE, 2004 (SPIE proceedings series; 5536). 134-145.

Oliver, E. C. \& Kockelmann, W. (2008). Report on moderator choice for IMAT. Unpublished report. ISIS, Didcot, UK.

Placzek, G. (1954). Phys. Rev. 93. 895-896.

Priesmeyer, H. G.; Stalder, M.; Vogel, S. C.; Meggers, K.; Bless, R. \& Trela, W. (1999). Textures and Microstructures 33. 173-185.

Santisteban, J. R.; Edwards, L.; Steuwer, A. \& Withers, P. J. (2001). J. Appl. Cryst. 34. 289-297.

Šaroun, J.; Pirling, T. \& Rogge, R. B. (2002). Appl. Phys. A 74. s1489-s1491.

Sears, V. (1992). Neutron News 3. 26-37.

Strobl, M. (2009). Nucl. Instrum. Methods Phys. Res., Sect. A 604. 646-652.

Strobl, M.; Hilger, A.; Boin, M.; Kardjilov, N.; Wimpory, R.; Clemens, D.; Mühlbauer, M.; Schillinger, B.; Wilpert, T.; Schulz, C.; Rolfs, K.; Davies, C. M.; ODowd, N.; Tiernan, P. \& Manke, I. (2010). Nucl. Instrum. Methods Phys. Res., Sect. A 651. 149-155.

Treimer, W.; Strobl, M.; Kardjilov, N.; Hilger, A. \& Manke, I. (2006). Appl. Phys. Lett. 89. 203504.

IUCr macros version 2.1.4: 2010/12/07 
Vogel, S. C. (2000). PhD thesis. Christian-Albrecht-University of Kiel, Germany.

Waller, I. (1923). Phys. Z. 19. 398-408.

Windsor, C. G. (1981). Pulsed Neutron Scattering. London: Taylor \& Francis.

Wyckoff, R. W. G. (1930). The Analytical Expression of the Results of the Theory of Spacegroups. Washington: Carnegie Institution of Washington.

\section{Synopsis}

A reusable program library to compute cold and thermal neutron cross sections and application examples for Bragg edge imaging are presented. 